\title{
IMPROVEMENT OF THE F-PERCEPTORY APPROACH THROUGH MANAGEMENT OF FUZZY COMPLEX GEOGRAPHIC OBJECTS
}

\author{
B. Khalfi a, b, C. de Runz ${ }^{\text {c, b }, ~ S . ~ F a i z ~}{ }^{a}$, H. Akdag ${ }^{b}$ \\ ${ }^{a}$ LTSIRS, Université de Tunis el Manar, Tunis, Tunisie \\ sami.faiz@insat.rnu.tn; khalfi@ai.univ-paris8.fr \\ ${ }^{\mathrm{b}}$ LIASD, Université Paris8, Paris, France \\ derunz@ai.univ-paris8.fr; akdag@ai.univ-paris8.fr \\ ${ }^{c}$ CReSTIC, Université de Reims Champagne-Ardenne, Reims, France \\ cyril.de-runz@univ-reims.fr
}

\section{Commission II, WG II/4}

KEY WORDS: Multiplicity, Imperfect data modeling, UML, fuzzy complex objects, F-Perceptory.

\begin{abstract}
:
In the real world, data is imperfect and in various ways such as imprecision, vagueness, uncertainty, ambiguity and inconsistency. For geographic data, the fuzzy aspect is mainly manifested in time, space and the function of objects and is due to a lack of precision. Therefore, the researchers in the domain emphasize the importance of modeling data structures in GIS but also their lack of adaptation to fuzzy data. The F-Perceptory approachh manages the modeling of imperfect geographic information with UML. This management is essential to maintain faithfulness to reality and to better guide the user in his decision-making. However, this approach does not manage fuzzy complex geographic objects. The latter presents a multiple object with similar or different geographic shapes. So, in this paper, we propose to improve the F-Perceptory approach by proposing to handle fuzzy complex geographic objects modeling. In a second step, we propose its transformation to the UML modeling.
\end{abstract}

\section{INTRODUCTION}

Any spatial analysis enables the geographer to identify the fundamental characteristics to the geographic data: the spatial character, temporal and the imprecise nature of this data. Then consider geographic information in its complex nature leads to adapt the classical representation, storage and processing methods and to take a specific consideration in models using this data.

Many methods have aimed to model the spatio-temporal information in a GIS, such as PERCEPTORY (Bédard, 1999), MECOSIG, POLLEN (Gayte et al. 1997) and MADS (Parent et al., 1997) and many others works have proposed solutions to represent and/or to modelize imprecise geographic data such as in (De Runz, 2008), (Miralles, 2006), (Parent et al., 1997) and (Zoghlami et al., 2014).

In this paper, we are interested in the conceptual level and especially for the F-Perceptory approach which is a reflection recently conducted by (Zoghlami et al., 2014) and (Zoghlami et al., 2015) for geographic databases modeling supporting the imprecision. Its current version presents some limits on its expression ability to describe complex and multiple geographic objects.

So, the present work aims to extend F-Perceptory with the managing of fuzzy complex geographic objects and then generating the corresponding UML model for each type of object complexity.

The remainder of this paper is organized as follows. In section 2 , we present the context and discuss the research issue. Section 3 presents geographic imprecision handling with F-Perceptory. Section 4 introduces our improvement for the modeling of fuzzy complex geographic objects and their mapping to UML Finally, conclusion and perspectives are made in section 5.

\section{CONTEXT AND RESEARCH ISSUE}

The problem of imperfection in spatial data models and essentially imprecise data is a current research question. To better ensure data quality in geographic databases and that data can be used in a valid way, it is necessary to take into account the imprecision of data and adapt data structures in GIS for this imperfect nature. Representations of data and information systems that ignore data imprecision appear less suitable for modeling of the real world.

Much effort has been made to represent imperfect data and some conceptual models have been extended to modelize this kind of data. Some have been developed and is presented in a technology, while others remain theoretical contributions. For example, CONGOO (Pantazis and Donnay, 1996) is a formalism for the design of object-oriented GIS. The authors of this formalism had reflected in the description of fuzzy concepts, but give no precise definition (Pantazis and Donnay, 1997).

POLLEN (Gayte et al., 1997) is an object design formalism for the environment, but it does not yet include the treatment of fuzzy geographic data.

As for the MADS method (application Modeling spatiotemporal dimension), it adopts an approach based on abstract data types for modeling of spatiality, temporality and topological relationships. The communication of (Shu et al., 2003) proposes 
an extension of MADS taking into account the fuzzy spatial and temporal concepts but has not exceeded the theoretical context.

Miralles in his thesis (Miralles, 2006) used the Model Driven Architecture (MDA) approach and proposed a UML profile for GIS modeling using pictograms. Miralles had reflected on fuzzy extensions, but his version has a limited ability to express imposed by the version of UML used. In addition, his work did not take into account the implications of this enrichment on database or on the exploitation of the latter through fuzzy queries.

F-Perceptory approach combines, in the one hand, Perceptory ${ }^{1}$ (Brodeur et al., 2000) that extends UML for modeling of GIS; Perceptory integrates a spatial PVL (Plug-in Visual Language) introduced by (Bédard, 1999) into UML by defining a set of geographic pictograms to identify spatial objects in database modeling. On the other hand, F-perceptory is based on fuzzy UML concepts that are proposed by (Ma, 2005) and (Wang, 2005). Fuzzy UML has been extended to include a graphical representation of fuzzy extensions for most UML concepts to complement the limited semantics of UML; it integrates extensions for fuzzy classes, fuzzy attributes and fuzzy relationships, but does not treat the fuzzy constraints. All these UML extensions are introduced in (Ma and Yan, 2007).

F-Perceptory defines a set of pictograms used to represent imprecise data. The pictograms are the same as those defined by Perceptory but with a dashed rectangular outline. A pictogram is used when the user wants to have the shape of an object class on the map. So, F-Perceptory attaches two types of information to the geographical object: on one side, graphical information (stereotypes) that gives an idea about the geometry of the spatial object and which is useful to illustrate its shape and its composition. On the other side, the textual information (attributes) to better describe the details of the object (properties, semantics). The combination of the two is essential to understand and provide a necessary wealth of expression for spatial data.

F-Perceptory does not consider, until now, all the possibilities of expression concerning the forms of spatial objects (object aggregation of fuzzy similar geometry, object aggregation of fuzzy multiple geometries, object with a facultative fuzzy geometry and object with fuzzy alternative geometries). FPerceptory omits the management of these types of fuzzy complex forms which makes the approach limited to simple objects defined by a unique stereotype.

Consequently, we propose to handle facultative objects which are represented by $(0,1)$ multiplicity, fuzzy objects with an alternative geometry, fuzzy multiple objects which aggregate $(0$, $\mathrm{N})$ or $(1, \mathrm{~N})$ similar shapes. We consider also fuzzy objects with complex aggregation geometry (different shapes). We manage, in a second step, the transformation modeling of these complex geometries from F-Perceptory to UML.

1 We call Perceptory the approach of representing by the PVLs (PictograpFs) the spatial and temporal objects in UML developed in the Perceptory Tool proposed by the Yvan Bedard's team (Bedard, 1999).

\section{GEOGRAPHIC IMPRECISION WITH F- PERCEPTORY}

The fuzzy aspect of data is mainly manifested in time, space and the function of objects and is due to a lack of precision. Geographic objects are often represented as points, lines or surfaces. This type of structure is well suited to objects whose contours are easy to locate, but for the representation of objects or phenomena whose boundaries are imprecise, this type of representation gives a few from perfect vision of reality.

\subsection{Geographic imprecision representation}

The fuzzy sets theory presented par (Zadeh, 1965), developed by (Kaufmann and Zadeh, 1973), and applied to the spatial analysis by (Ponsard, 1980), is embedded in many approaches to deal with imperfect information essentially for the quantification of imprecision.

In the sets theory, each element e whether it belongs entirely to a subset $E$ of a given repository or does not belong. If this element $e$ is assigned a membership function to $E$, the latter can take usually 0 for non-membership and 1 of belonging to $E$. However, the theory of fuzzy sets (Zadeh, 1965), chooses to represent e that has partial membership in a set and to assign to it a membership function that takes values in the interval $[0,1]$. A fuzzy set is a set defined using such a membership function and a fuzzy element is an element whose value belongs to a fuzzy set.

The F-Perceptory approach is based on the fuzzy sets theory, so, we present the following definitions concerning fuzzy data (Zadeh, 1965), which are necessary to understand the concepts discussed in the rest of this paper:

Definition1: Let $\boldsymbol{U}$ be a universe of discourse and $\mathbf{A}$ be a fuzzy set in $\mathbf{U}$ and $\boldsymbol{\mu} \mathbf{A}$ a membership function. $\mathbf{A}$ is formally defined by its membership function $\boldsymbol{\mu} \mathbf{A}$.

Definition2: The membership function is defined for $\boldsymbol{A}$ as follows: $\boldsymbol{\mu A}: \boldsymbol{U} \rightarrow[\mathbf{0}, \mathbf{1}]$. Here $\boldsymbol{\mu} \boldsymbol{A}(\boldsymbol{x})$ for each $\mathrm{x} \in \boldsymbol{U}$ denotes the membership degree of $\boldsymbol{x}$ in the fuzzy set $\boldsymbol{A}$.

Definition3: For any set $\mathbf{E}$, an $\boldsymbol{\alpha}$-cut $\mathbf{A} \boldsymbol{\alpha}$ of a fuzzy set $\mathbf{A}$ defined on $\mathbf{E}$ is the set of elements for which the value of the membership function $\boldsymbol{\mu} \mathbf{A}$ of $\mathbf{A}$ is greater to $\boldsymbol{\alpha}$, that is to say:

$$
\mathrm{A} \alpha=\{\mathrm{x} \in \mathrm{E}, \mu \mathrm{A}(\mathrm{x}) \geq \alpha\} \text { avec } \alpha>0 .
$$

Definition4: A fuzzy set A is connected if and only if for all $\alpha$ $(0 \leq \alpha \leq 1) A \alpha$ is connected. A $\alpha$ is connected if and only if we cannot make a bipartition of A $\alpha$ with two non-empty open spaces. That is to say, for each pair of lines belonging to $A \alpha$, there is a path connecting them included in A $\alpha$.

Definition5: $\mathbf{A}$ is said normalized if and only if $\mathbf{H}(\mathbf{A})=1$. The height of $\mathrm{A}(\mathrm{H}(\mathrm{A}))$ is the maximum value of the membership function $\boldsymbol{\mu} \mathbf{A}$.

$$
\mathrm{H}(\mathrm{A})=\max _{\mathrm{x}} \in \mathrm{U}(\mu \mathrm{A}(\mathrm{x}))
$$




\subsection{Geographic imprecision modeling in F-Perceptory}

Spatial imprecision is modeled in F-Perceptory (see figure 1) by using of fuzzy pictograms. Pictograms present Fuzzy geometry which introduces spatial objects whose boundaries are not defined properly.

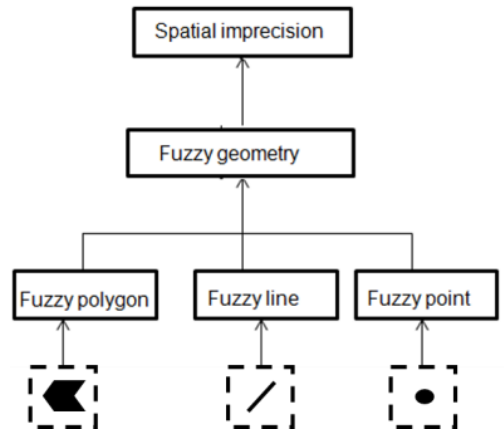

Figure 1. Spatial imprecision in F-Perceptory

The approach focuses on three simple spatial shapes: Fuzzy point (0-dimension), Fuzzy line (1-dimension), and Fuzzy polygon (2-dimension).

0 -dimension pictogram is used generally to represent objects when the exact detail of the contour is not important at a given scale. A traffic roundabout can be represented as a point shape. For a fuzzy traffic roundabout, we consider the fuzzy point shape.

1-dimension pictogram is used to represent a path between two ends. For instance, we can associate the line shape for a road segment or a river. A fuzzy river is represented as a fuzzy line.

2-dimension pictogram is used to represent large sized areas. The limits of cities, parks, buildings are usually represented by polygons when the scale is sufficiently high to be able to distinguish their areas. For instance, fuzzy parks are represented with a fuzzy polygon pictogram.

\begin{tabular}{|l|l|c|}
\hline $\begin{array}{l}\text { Geometry } \\
\text { type }\end{array}$ & Meaning & Example \\
\hline Simple & $\begin{array}{l}\text { Object represented by a point } \\
\text { shape (0 dimension). }\end{array}$ & \\
\cline { 2 - 3 } & $\begin{array}{l}\text { Object represented by a line } \\
\text { shape (1 dimension). }\end{array}$ & \\
\cline { 2 - 3 } & $\begin{array}{l}\text { Object represented by a } \\
\text { polygon shape (2 dimension) }\end{array}$ & \\
\hline Facultative & $\begin{array}{l}\text { Object represented by a } \\
\text { simple shape with multiplicity } \\
\text { (0,1) }\end{array}$ & 0,1 \\
\hline Alternative & $\begin{array}{l}\text { Object represented by one or } \\
\text { the other shape. }\end{array}$ & \\
\hline Multiple & $\begin{array}{l}\text { Object represented by several } \\
\text { shapes but only one is used in } \\
\text { a representation. }\end{array}$ & $\begin{array}{l}\text { Object represented by more } \\
\text { than one shape. }\end{array}$ \\
\hline Complex & $\vdots$ \\
\hline
\end{tabular}

Table 1: F-Perceptory types of spatial geometries

Based on primitive shapes, the specification of F-Perceptory introduced five types of geometries to represent the real world objects. The possible geometries are summarized in table 1 .

According to constraints that can be defined on geographic proprieties of spatial objects (shape, composition, size) or on the scale used (large or limited) to represent these objects, we can distinguish the following types of geometries:

- The facultative geometry, so, according to a condition on the geometric properties of the simple spatial object, this latter may or may not be mapped.

- The alternative geometry where a spatial object can be represented by one of two possible shapes according also to a condition on its geometric properties. The roads can be represented as polygons when the scale is high and as lines when the scale is small enough.

- The multiple geometry where a spatial object may be represented by one of two possible shapes according to the scale taken. For example, the cities on a world map can be described in the form of points while a regional map would use a polygonal representation of cities.

- The complex geometry where a spatial object is represented by an aggregation of different simple shapes. The object is simultaneously represented by several geometric primitives. For example, a hydrographic network is composed of several rivers (line representation) and lakes (polygon representation).

Although F-Perceptory modelizes multiple and complex geometries, it did not propose their mapping principles to UML. For UML conversion of fuzzy model, F-Perceptory still limited to model and map only simple geometries (fuzzy point, fuzzy line and fuzzy polygon). So, it is useful and in some cases, necessary to consider complex cases in modeling. For completeness, we propose to extend the approach by adding the modeling of complex geometries and their mapping. As a result, we consider the modeling of facultative, alternative, multiple and complex geometry and we add also the case of the collection of shapes. Despite the multiple geometry, the collection presents the case in which the spatial object is an aggregation of one simple shape type. For example, a street in the real world might consist of several lines, each representing a road segment with its different attributes.

\begin{tabular}{|l|l|c|}
\hline Geometry type & Meaning & Example \\
\hline $\begin{array}{l}\text { Collection of } \\
\text { shapes }\end{array}$ & $\begin{array}{l}\text { Object represented by an } \\
\text { aggregation of one simple shape } \\
\text { type. }\end{array}$ & $1, \mathrm{~N}$ \\
\hline
\end{tabular}

Table 2: Collection of shapes: another type of complex geometry

\subsection{Simple fuzzy geometry mapping to UML}

Contrary to the modeling of non fuzzy classes, where every instance of those classes will have exactly one instance of such geometry, the modeling of fuzzy geographic classes means that every instance of those classes will have a set of instances of such geometry with a degree of possibility for each one.

We give, in this section, the mapping of a simple geographic class that has the polygon geometry. The mapping can be generalized to all forms of geometry (point and line). The figure 
2 shows the fuzzy polygon modeling in UML as proposed in (Zoghlami et al., 2011).

In this figure, the fuzzy polygon class $\boldsymbol{C s - p o l y g o n}$ is represented by a UML class composed of n classes of FormImperfection. So, each spatial polygon object is composed of $n$ polygon geometries with different membership degrees, which correspond to $\alpha$-cuts on a fuzzy set of polygon geometries.

The FormImperfection class is a spatial class with a geometric attribute (geometry) and a degree associated with it (degree). The geometries role of the composition relationship provides the set of geometries that compose the fuzzy spatial object, and the spatialObject role refers to the object of such geometric imperfection.

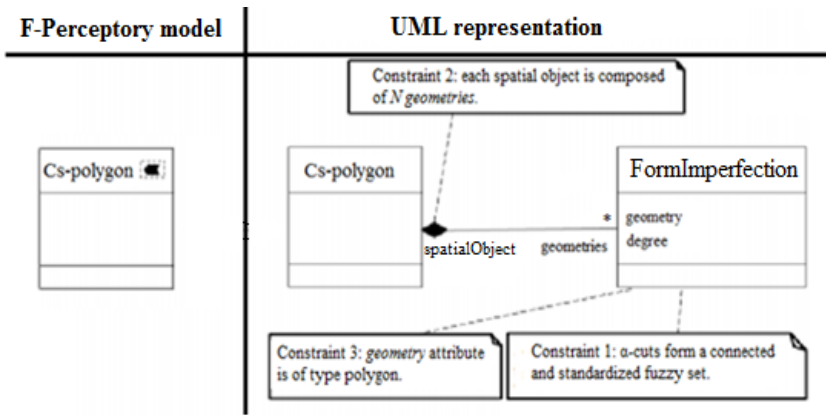

Figure 2. Transformation of a simple fuzzy polygon to UML

Three main constraints must be respected in this modeling according to (Zoghlami et al., 2014). The first constraint is to verify that the $\alpha$-cuts (definition 3 ) form a connected (definition 4) and normalized fuzzy set (definition5), that means that: 1) regardless of the G1 geometry with a degree $\mathrm{d} 1$, all geometries about our fuzzy set having a higher degree than $\mathrm{d} 1$ are included in $\mathrm{G} 1$; 2) forms are related geometric shapes; 3) maximum degree is equal to 1 . For the second constraint, it is necessary to verify that each spatial object of the Cs-polygon class is composed of $n$ geometries (with $n=$ number of $\alpha$-cuts). The last constraint is to verify that the geometry attribute is of type polygon.

The same process is applied to other types of simple spatial entity (point and line), we present in this paragraph the essential aspects of their mapping to UML:

A fuzzy line class is represented by a line class that is composed of $\mathrm{n}$ geometries represented by the spatial class FormImperfection. Each geometry is a polygon and has a degree of possibility. If the degree of possibility is equal to 1 , the geometry type is line.

- A fuzzy point class is represented by a point class that is composed of $\mathrm{n}$ geometries represented by the spatial class FormImperfection. Each geometry is a polygon and has a degree of possibility. The geometry type is point when the degree of possibility is equal to 1 .

\section{MANAGEMENT OF FUZZY COMPLEX GEOGRAPHIC OBJECTS}

\subsection{Fuzzy facultative geometry}

\subsubsection{Definition}

Fuzzy facultative geometry presents a geographic object with the multiplicity $(0,1)$. It is used for simple geometries to indicate whether the object is possibly mapped according to conditions.

We refer to the example in figure 3 witch models a fuzzy polygon class with multiplicity $(0,1)$ named House. So, a House object has a surface geometry (polygon), it will be mapped only where the area is greater than $500 \mathrm{~m}^{2}$, so we indicate the multiplicity 1 , otherwise, it is not mapped and we indicate the multiplicity 0 .

\begin{tabular}{|l|}
\hline House 0,1 \\
\hline $\begin{array}{l}\text { address } \\
\text { ConstructionDate } \\
\text { tloorNum }\end{array}$ \\
\hline
\end{tabular}

Figure 3. Example of fuzzy facultative geometry

\subsubsection{Mapping to UML}

The UML modeling of a fuzzy facultative geometry is shown in figure 4. We propose here to transform the House class.

The UML modeling of the fuzzy entity <House> uses three classes: (1) the class <House> to introduce all House objects that are manipulated. (2) The <IntermediatePolygon> to present fuzzy House objects that satisfy the condition. (3) $<$ FormImperfection> class that shows all the imperfections of a fuzzy object.

The association relationship between <House> class and $<$ IntermediatePolygon> class presents the multiplicity $(0,1)$ of fuzzy polygon for each House instance. If the House occurrence has the area greater than $500 \mathrm{~m}^{2}$, then it will be mapped and has one occurrence of a fuzzy polygon; otherwise, it will be not mapped and has no occurrence of fuzzy polygon, so no IntermediatePolygon occurrence.

The three constraints defined in the mapping of fuzzy simple class are also applied to the facultative geometry on House class and FormImperfection class.

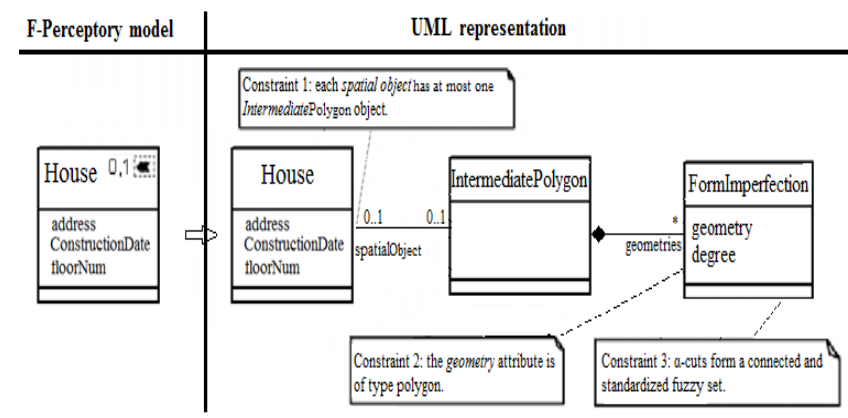

Figure 4. Fuzzy facultative polygon mapping 


\subsection{Fuzzy collection geometry}

\subsubsection{Definition}

The collection geometry called simple aggregate geometry in (Bédard, 1999) is used to represent geometry that aggregates several similar shapes (having the same dimension). Instead of using multiple pictograms of the same shape, we add the $(0,1)$ or $(1, \mathrm{~N})$ multiplicity next to the pictogram. For example, road networks are composed of several linear segments, consequently the RoadNetwork class has the 1-dimension pictogram (fuzzy line) followed by a $(1, \mathrm{~N})$ multiplicity (figure5).

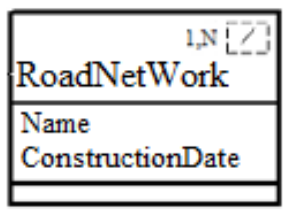

Figure 5. Example of fuzzy collection geometry

\subsubsection{Mapping to UML}

To manage the aggregation of the object, we add a class that will represent the set of its primitive shapes. Each primitive will be fuzzy and be modeled in UML as a simple fuzzy object (referring to figure 2).

The UML modeling of a simple aggregate geometry is shown in figure 6. We propose here to transform the RoadNetWork class that aggregates $(1, \mathrm{~N})$ line shapes. It is represented in UML by the RoadNetWork class that is related to IntermediateLine class with an association relationship. This relationship presents the multiplicity $(1, \mathrm{~N})$ of lines for each RoadNetWork instance. So, the IntermediateLine class will present the set of fuzzy line objects that will compose each RoadNetWork instance (Constraint 1).

Since RoadNetWork is fuzzy, so the set of IntermediateLine objects is fuzzy too. Each object in this set will be composed of $\mathrm{n}$ line geometries (Constraint 2) with different membership degrees. FormImperfection class shows all geometries with their degrees of possibility.

The three constraints defined in the mapping of fuzzy simple class are also applied to the collection geometry on RoadNetWork class and FormImperfection class.

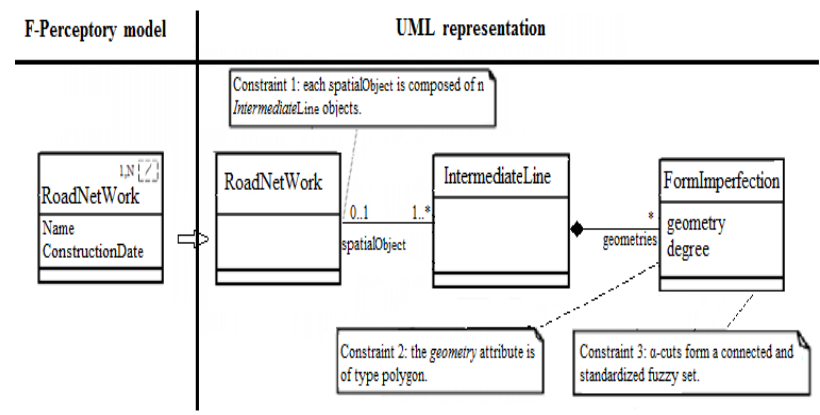

Figure 6. Fuzzy simple aggregate polygon mapping
The same process is applied to other types of multiple spatial entity (point and polygon); we present in this paragraph the essential aspects of their mapping to UML:

- A multiple fuzzy line class is represented by a line class that has a set of IntermediateLine class. Each IntermediateLine object has $(0, \mathrm{~N})$ geometries represented by the FormImperfection class.

- A multiple fuzzy polygon class is represented by a polygon class that has a set of IntermediatePolygon class. Each IntermediatePolygon object has $(0, \mathrm{~N})$ geometries represented by the FormImperfection class.

\subsection{Fuzzy complex geometry}

\subsubsection{Definition}

Fuzzy complex geometry is used to represent a fuzzy geometry where the aggregate is composed of multiple shapes with different dimensions. This can be depicted by inserting the appropriate pictograms. The complex aggregate geometry has the default multiplicity $(1, \mathrm{~N})$ that will not be expressed.

The example of figure 7 models a Hydrographic Network, which is an aggregation of several fuzzy lines that present rivers and fuzzy polygons that present lakes. So, it is modeled by a fuzzy line and fuzzy polygon pictograms.

We limit the number of the combination of shapes to two pictograms.

\begin{tabular}{|l|}
\hline $\begin{array}{l}\text { Hydrographic } \\
\text { Network }\end{array}$ \\
\hline $\begin{array}{l}\text { Name } \\
\text { ConstructionDate }\end{array}$ \\
\hline
\end{tabular}

Figure 7. Example of fuzzy complex geometry

\subsubsection{Mapping to UML}

We propose here, to transform the example of a Hydrographic Network (see figure 8).

Each occurrence of <HydrographicNetwork> is composed of $(1, \mathrm{~N})$ fuzzy polygons and $(1, \mathrm{~N})$ fuzzy lines. So, in UML modeling, we define the class IntermediatePolygon for the set of fuzzy polygon objects and IntermediateLine for the set of fuzzy line objects.

Each polygon object is fuzzy and each line object is fuzzy too. So, we need to define the FormImperfection class that stores the several fuzzy geometries.

The FormImperfection class is a common class among all geometries. The constraints applied on FormImperfection class are the same, although they are not shown on figure 8 . 


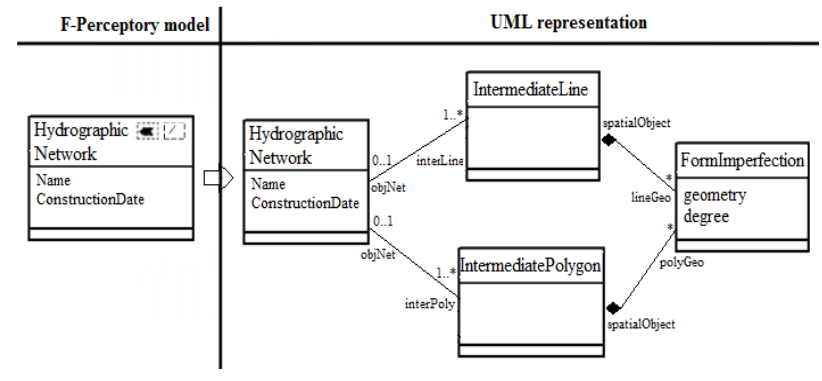

Figure 8. Fuzzy complex geometry mapping

The three constraints defined in the mapping of fuzzy simple class are also applied to the complex geometry on HydrographicNetwork class and FormImperfection class.

\subsection{Fuzzy alternative geometry}

\subsubsection{Definition}

The alternative geometry is defined by Bedard in (Bedard, 1999) when each occurrence of a class of objects has a shape of one dimension or the other, but never both. It represents the exclusive 'OR' between the two possible shapes.

Fuzzy alternative geometry introduced in (Zoghlami et al., 2011), is defined when the object has an imprecise geometric shape in both cases. Then, it is represented by the concatenation of the possible pictograms (see figure 9).

For example, when a building is represented by either a fuzzy point (if its area is smaller than 1 hectare) or by a fuzzy polygon (if its area is larger than 1 hectare), then it has the 0-dimension and 2-dimenssion pictograms adjacent to each other (see figure 9). There is no space between the pictograms and their ordering has no meaning.

\begin{tabular}{|l|}
\hline Building $\quad-\infty$ \\
\hline Name \\
ConstructionDate \\
\hline
\end{tabular}

Figure 9. Example of fuzzy alternative geometry

\subsubsection{Mapping to UML}

We propose to transform the example of Building class (see figure10). Each Building occurrence can be mapped either with a fuzzy polygon instance, or with a fuzzy line instance according to some conditions.

So, in UML modeling, we define the class <IntermediatePolygon> for fuzzy polygon objects and $<$ IntermediatePoint> for fuzzy point objects. We must define the $\{\mathrm{XOR}\}$ constraint to assure that each building occurrence will be presented by one spatial object not both. Each polygon object is fuzzy and each point object is fuzzy too, so we need to define the FormImperfection class that stores the several fuzzy geometries.

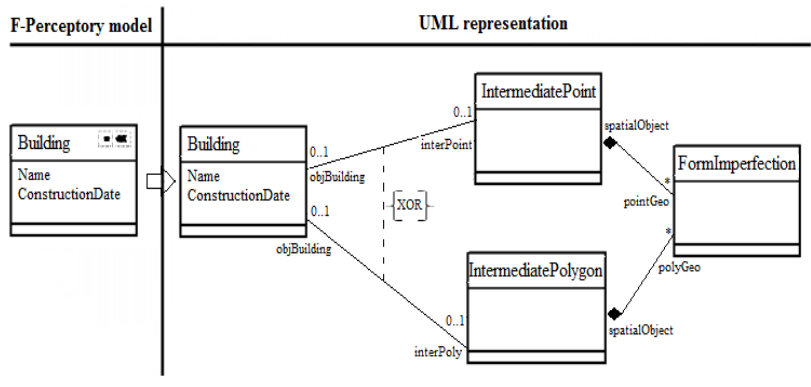

Figure 10. Fuzzy alternative geometry mapping

In addition to the $\{\mathrm{XOR}\}$ constraint defined for the association relationships between Building class and intermediate class, the three constraints defined in the mapping of the fuzzy simple class still applied to the alternative geometry on Building class and FormImperfection class.

\subsection{Fuzzy multiple geometry}

\subsubsection{Definition}

Multiple geometry is required for multiple representations at multiple scales (Bedard, 1999). It is similar to the alternative geometry but with the scale constraint. For example, a road segment (see figure 11) may have a surface geometry on a large scale and linear geometry on a small scale. The object with multiple geometry is represented by several geometries but of which only one is used in a map display or in a spatial query.

Perceptory modelizes the multiple geometry as the concatenation of pictograms. Perceptory does not limit the number of possible geometries. However, the authors of Fperceptory choose to represent the possible pictograms as superimposed geometries and they limited the number of pictograms to two.

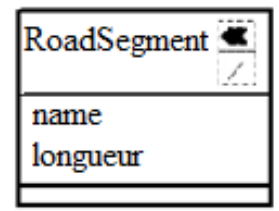

Figure 11. Example of fuzzy multiple geometry

\subsubsection{Mapping to UML}

The UML modeling of multiple geometry passes through the same transformation principles of alternative geometry.

For the example of RoadSegment, we define the class <IntermediatePolygon> for fuzzy polygon objects and $<$ IntermediateLine> for fuzzy line objects. We define also the $\{\mathrm{XOR}\}$ constraint to assure that each RoadSegment occurrence will be presented by one spatial object not both for a given scale. So, at a global scale, each RoadSegment occurrence will be presented by one fuzzy line object (represented by the IntermediateLine class). However, at a local scale, it will be presented by one fuzzy polygon object (represented by the IntermediatePolygon class). 


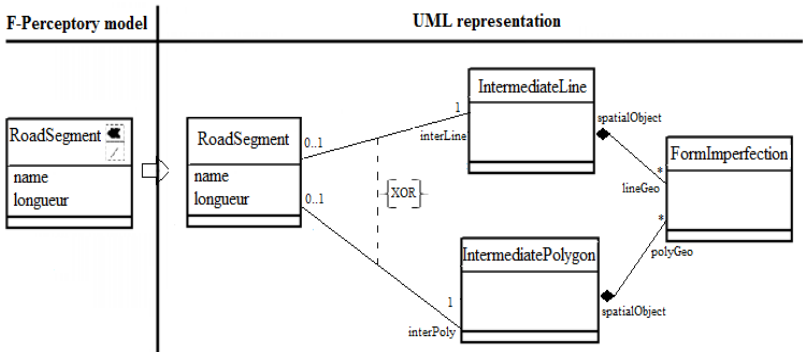

Figure 12. Fuzzy multiple geometry mapping

\section{CONCLUSION AND PERSPECTIVES}

F-Perceptory is a modeling approach for fuzzy geographic data based on stereotype concept and UML language. It defines the conceptual model for databases. It describes fuzzy entities, fuzzy attributes (characteristics and descriptions of entities) and relationships between entities.

However, the approach has some weakness, so, we proposed to enhance it. The new version, proposed in this paper, allows the user to present a fuzzy model with all possible types of fuzzy objects (simple and complex).

Then, we proposed the generation of the corresponding UML representation. We manage here the mapping from a fuzzy model to a UML model by the definition of the necessary constraints applied to classes, objects, attributes and relationships.

As a second step, we plan the enrichment of F-Perceptory through the development of fuzzy relationships and define their mappings to UML and their related constraints of course.

We propose also to develop an automatic information treatment process that modelizes fuzzy geographic information system. So, it is to propose a module developed and integrated under an existing CASE tool. This module offers the user an FPerceptory palette to define his fuzzy schema, the possibility to map from fuzzy to UML (conceptual model), and to map from UML to logic schema to enable the implementation of the conceptual model in a relational model and from logic model to the physical schema where he obtains the script code of the database creation.

\section{REFERENCES}

Bédard, Y., 1999. Visual modelling of spatial databases : Towards spatial PVL and UML. Geomatica, 53, pp. 169-186.

Brodeur, J., Bédard, Y. and Proulx, M. J., 2000. Modelling Geospatial Application Database. Proceedings of 8th ACM International Symposium on Information and Knowledge, New York, NY, USA, pp. 39-46.

Gayte, O., Libourel, T., Cheylan, J.-P. and Lardon, S., 1997. Conception des systemes d'information sur l'environnement. Paris: Hermès.
Kaufmann, A. and Zadeh, L. A., 1973. Introduction à la théorie des sous-ensembles flous. Paris : Masson.

Ma, Z., 2005. Fuzzy information modeling with the UML. Advances in fuzzy object-oriented databases : modeling and applications, IGI Global, pp. 153-176.

Ma, Z. and Yan, L., 2007. Fuzzy XML data modeling with the UML and relational data models. Data and Knowledge Engineering, 63(3), pp. 970-994.

Ma, Z. and Yan, 1., 2010. A literature overview of fuzzy conceptual data modeling. Journal of information Science and Engineering, 26(1), pp.427-441.

Miralles, A., 2006. Ingénierie des modèles pour les applications environnementales. Paris: Phd Thesis, University of Montpellier 2.

Pantazis, D. N. and Donnay, J.-P., 1996. La conception de SIG : méthode et formalisme. Paris: Hermés.

Pantazis, D. N. and Donnay, J.-P., 1997. Objets géographiques à limites indéterminés Modélisation et intégration dans un modèle conceptuel de données. Revue Internationale de Géomatique, 7(2), pp. 159-186.

Parent, C., Spaccapietra, S., Zimnyi, E., Donini, P., Plazanet, C., Vangenot, C., Rognon, N. and Crausaz, P.-A., 1997. MADS: un modèle conceptuel pour des applications spatiotemporelles. Revue Internationale de Geomatique, 7(3/4), pp. 317-352.

Ponsard, C., 1980. Fuzzy economic spaces, Université de Dijon: Document de travail de l'Insititut de Mathématiques Ecomoniques.

Shu, H., Spaccapietra, S., Parent, C. and Sedas, D., 2003. Uncertainty of Geographic Information and its Support in MADS. International Symposium on Spatial Data Quality (ISSDQ), Hong Kong, China.

Wang, L., 2005. Fuzzy UML, Karlsruhe: University of Karlsruhe.

Zadeh, L. A., 1965. Fuzzy sets. Information and Control, 8(3), pp. 338-353.

Zadeh, L. A., 1978. Fuzzy Sets as a Basis for a Theory of Possibility. Fuzzy Sets and Systems, 1(1), pp. 3-28.

Zoghlami, A., de Runz, C. and Akdag, H., 2014. Handling Imprecise Data in Geographic Databases. Encyclopedia of Information Science and Technology, IGI Global, chapitre 172, Third Edition, pp. 1785-1799.

Zoghlami, A., de Runz, C. and Akdag, H., 2015. F-Perceptory: an approach for handling fuzziness of spatiotemporal data in geographical databases. International Journal of Spatial, Temporal and Multimedia Information Systems (IJSTMIS), Inderscience, in press.

Zoghlami, A., de Runz, C., Akdag, H., Zaghdoud, M. and Ben Ghezala, H., 2011. Handling imperfect spatiotemporal information from the conceptual modeling to database structures. International Symposium on Spatial Data Quality (ISSDQ), Coimbra, Portugal, pp. 165-170. 